\title{
Buckling by General Instability of Cylindrical Components of Deep Sea Submersibles
}

\author{
C.T.F. Ross ${ }^{1 \mathrm{a}}$, K. O. Okoto ${ }^{1 \mathrm{~b}}$ and A.P.F. Little ${ }^{1 \mathrm{c}}$
}

\section{${ }^{1}$ University of Portsmouth, Department of Mechanical Engineering, Anglesea Road, Portsmouth, PO1 3DJ., UK.}

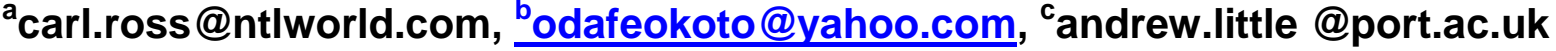 \\ Keywords: General instability, ring-stiffened cylinders, external pressure, finite \\ elements.
}

\begin{abstract}
.
This paper reports on theoretical and experimental investigations into the buckling characteristics of a series of six ring-stiffened circular cylinders that experienced general instability when subjected to external hydrostatic pressure. Each study used between 3-5 designs with the same internal and external diameters, but with different numbers and sizes of ring-stiffeners. Four used designs that were machined to a high degree of precision from steel, while the other two were machined from aluminium alloy.

The theoretical investigations focused on obtaining critical buckling pressure values, namely $\mathrm{P}_{\mathrm{cr}}$, for each design from the well-known Kendrick's Part I and Part III theories, together with an ANSYS finite element prediction. The thinness ratio $\lambda^{1}$, which was originally derived by the senior author, was calculated together with a dimensionless quantity called the plastic knockdown factor (PKD), for each model. The plastic knockdown factor was calculated by dividing the theoretical critical buckling pressures $\mathrm{P}_{\mathrm{cr}}$, by the experimental buckling pressures $\mathrm{P}_{\text {exp. }}$ The thinness ratio was used because vessels such as these, which have small but significant random out-of-circularity, defy "exact" theoretical analysis and it is because of this that the design charts were produced.

Three design charts were constructed by plotting the reciprocal of the thinness ratio $\left(1 / \lambda^{1}\right)$ against the plastic knockdown factor $\left(\mathrm{P}_{\mathrm{cr}} / \mathrm{P}_{\mathrm{exp}}\right)$, using results from Kendrick Part I, Kendrick Part III, and ANSYS. Comparison of the results obtained using Kendrick's theories and experimentally obtained results was good.
\end{abstract}

\section{Introduction}

In totality, the world's oceanic waters cover about $71 \%$ of the Earth's surface. Nearly half of these waters are over 3,000 meters $(9,800 \mathrm{ft})$ deep [1,2], but only about one percent of the Earth's Oceans have been explored. Considerable research has been carried out in the oceans over the last 40 years, to understand processes responsible for plate tectonics and ocean chemistry, as well as the origins of life and mechanisms for speciation. Even more is yet to be discovered! It is evident that in the twenty first century, man will exploit the ocean deeps for oil, gas, minerals and precious metals under more challenging environments.

The study of the strength of ring-reinforced circular cylinders under uniform external pressure is very important in the design of pressure vessels that can be used to build submarines or submersibles. Scientists have been researching for faster and more efficient methods of determining design parameters for ring-stiffened circular cylinders and this is the focus of this work. 


\section{Background}

A structural system is formed by a structure and the loading acting on it. There are two main properties that make a structure withstand loads, which are the constitutive material and the geometric shape. Buckling is a process by which a structure cannot withstand load with its original geometry and material. Two forms of buckling are shown in Figs. $1 \& 2$, namely shell instability (or non-symmetric bifurcation buckling) and general instability, where the entire ring shell combination buckles in its flank.

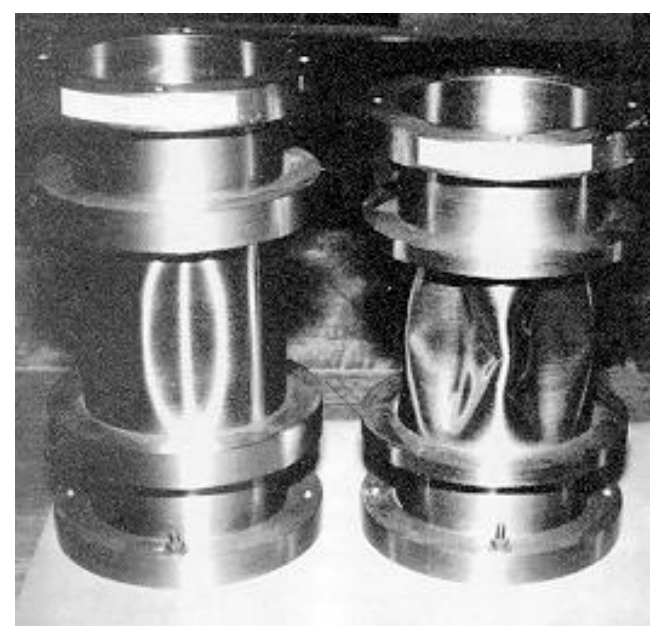

Figure 1: Shell instability.

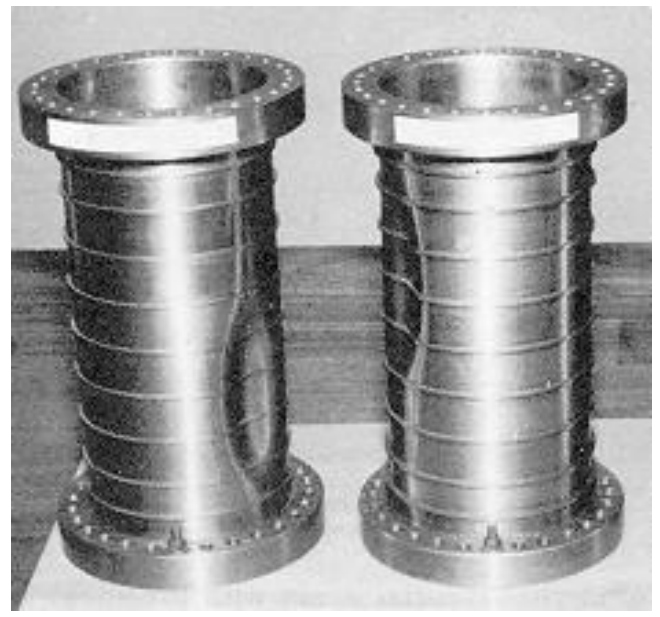

Figure 2: General instability.

Very often, the external pressure required to cause the shell instability of a thin-walled circular cylinder or cone is only a fraction of that necessary to cause axisymmetric yield for a similar vessel under uniform internal pressure. In the case of axisymmetric failure, the cylinder implodes, keeping its circular form during failure. One way of improving the structural efficiency of these vessels is to stiffen them with suitably sized ring-stiffeners either internally or externally, or both. General instability is the process where the entire ring-shell combination of a circular cylinder or cone buckles between adjacent bulkheads, as shown in Fig. 2 This mode of failure was first identified by Tokugawa [3]. A major breakthrough on the general instability of ring-stiffened circular cylinders was due to Kendrick [4-8]. His solutions, which assumed simply supported edges, were based on the Rayleigh-Ritz method.

Kendrick derived an expression for the total strain energy and potential and assumed a simple trignometrical series, which he substituted into his strain energy expressions, to obtain a buckling pressure. Ross [1] developed two computer programs namely; KENDRIC1 and KENDPT3 to calculate the critical buckling pressure due to general instability automatically. KENDRIC1 and KENDPT3 employ Kendrick's Part I and Part III theories, respectively. Windenburg and Trilling [9] introduced a thinness ratio " $\lambda$ " to consider the effects of out-of-roundness on un-stiffened cylinders and Ross [10] modified this to cater for the plastic general instability of ring-stiffened cylinders; he called this his thinness ratio " $\lambda$ "”.

\section{Previous Experimental Investigations.}

The work in this paper was based mostly on past experiments carried out by Ross et al [1, 11, 12]; most of the models were made from EN1A mild steel and were machined to specification and checked for dimensional tolerance. The models were tested to destruction in tanks similar to that shown in Fig. 3. From Fig. 3, it can be seen that the tank was open at the top, so that the strain gauges could easily be attached to the inside surface of each cylinder. 


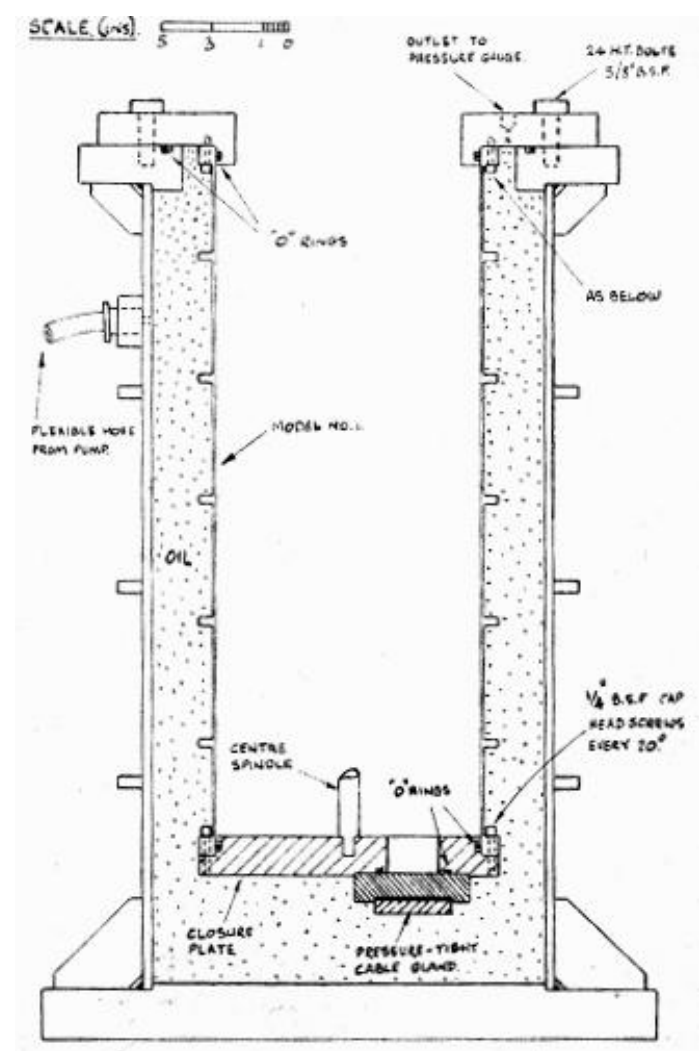

Figure 3: Test tank with a typical ring-stiffened model

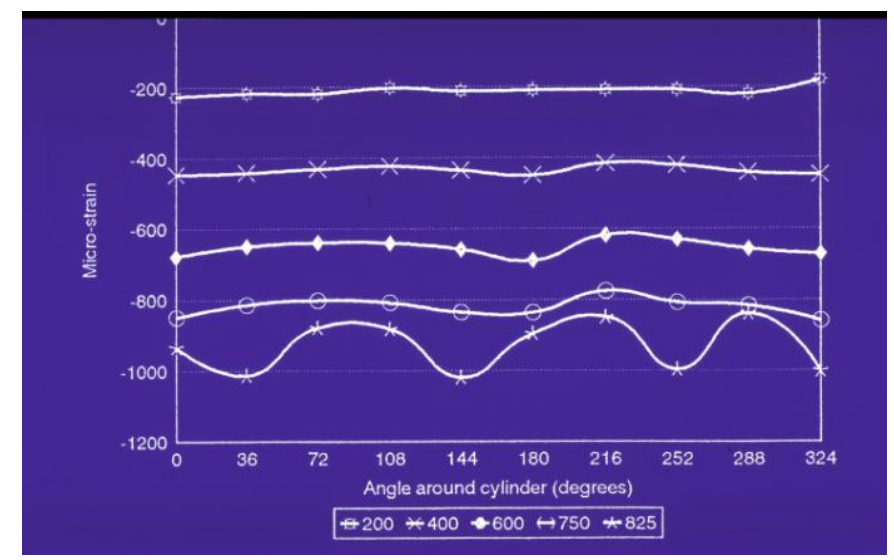

Figure 4: Circumferential strain plots at various pressures

Eleven circumferential strain gauges were connected to the inside of each cylinder. Ten of the strain gauges were fixed at points of equal spacing along the circumference of each cylinder, at its mid-span. Strain measurements from the gauges were used to determine the circumferential wave pattern of the specimen while buckling. An eleventh gauge was placed at mid-span to measure longitudinal strain. The models were tested to destruction under uniform external pressure, where the buckling pressures, namely $\mathrm{P}_{\text {exp }}$ were noted. A typical circumferential strain plot is shown in Fig. 4, where it can be seen that as the external pressure was increased, the magnitudes of the circumferential strains grew with increasing external pressure, eventually making a distinct wave pattern prior to collapse. The vertical axis of Fig. 4 was the circumferential strain and the horizontal axis was the azimuthal angle in degrees. The numbers in the box at the bottom of the table represent the various values of applied external pressure in Psi.

\section{Theoretical Investigations.}

The theoretical investigations used analytical and finite element theories to study the inelastic general instability of ring-stiffened circular cylinders. Investigations were carried out on the buckling characteristics of a number of ring-stiffened cylindrical models, subjected to external hydrostatic pressure. Each of the models failed by general instability when they were experimentally tested to failure, by subjecting them to external hydrostatic pressure. $\mathrm{P}_{\mathrm{cr}}$ was calculated from the Kendrick Programs and from ANSYS using the Shell 93 and Beam 44 elements. Figure 5 shows a screen dump of a typical ANSYS model with ring stiffeners. 


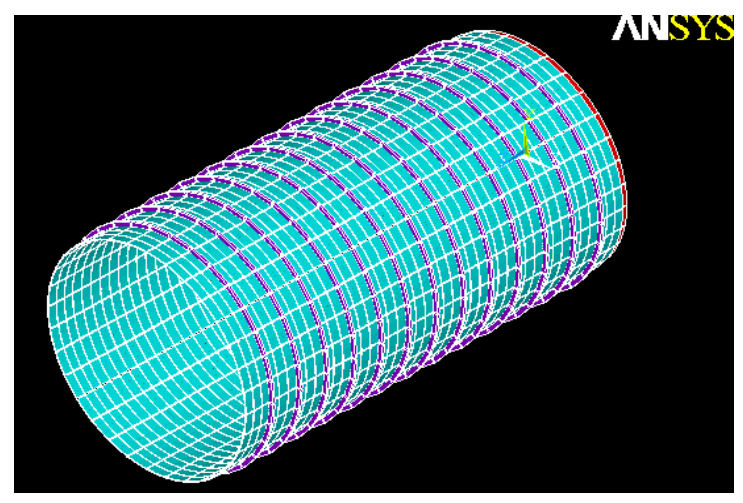

Figure 5: Reynolds' ring-stiffened cylinder, created with ANSYS.

\section{The Design Charts.}

In Figures 6 to 8, the data of the series of the six model designs that were destroyed, were obtained from the references given below in square brackets:

(1) Models P4, P5 \& P6 from Ross [1]; (2) Models 4,5 \& 6 from Ross [1]; (3) Models P1 to P2/A from Ross [1]; (4) PH series of models from Ross [12]; (5) Reynolds' Series of models from [14]; (6) Seleim's Series of models from [13].

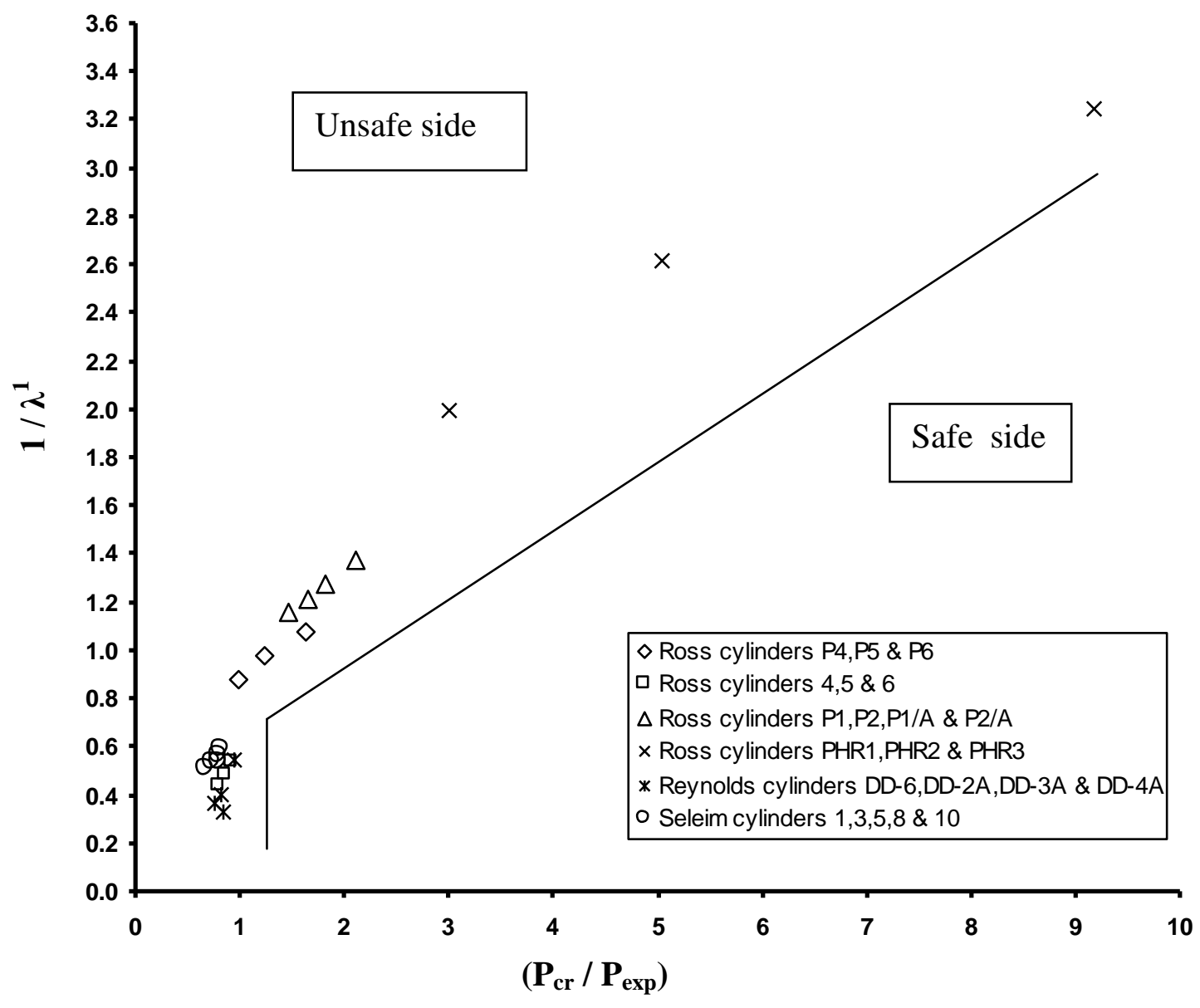

Figure 6: Design chart for the general instability of machined ring-stiffened cylinders based on Kendrick's Part I theory 
The data from the above investigations were then collated and presented in a tabular form and the results were used to calculate the two dimensionless parameters of the models for the final design charts, namely: the reciprocal of thinness ratio $=1 / \lambda^{1}$ and the plastic knockdown $=\mathrm{P}_{\mathrm{cr}} / \mathrm{P}_{\mathrm{exp}}$. The plastic knockdown (PKD) for all models was calculated using the theoretical buckling pressures from Kendrick Part I, Kendrick Part III and ANSYS and the experimentally derived failure pressures. The design charts were then constructed by plotting the reciprocal of the thinness ratio against the plastic knockdown factors [1,12-14]. Design charts based on Kendrick's Part I theory; Kendrick's Part III theory and finite element analyses using ANSYS are shown in Figures 6, 7 \& 8 , respectively, where the so-called safe side is to the right and below the lines.

\section{Discussion.}

Theoretical buckling pressures were calculated using Kendrick's Part I and Part III theories, and by simulation with ANSYS. Buckling pressures from Kendrick Part III is seen to be about $2 \%$ less than that calculated from Kendrick Part I. While both theories assumed simply supported edges, Kendrick Part I shows a buckling configuration where the ring-stiffened cylinder fails completely by general instability, while Kendrick Part III shows a buckling configuration where the ring-stiffened cylinder suffers additional displacements between the ring-stiffeners. As a result of this, this difference will be expected, with buckling pressures from Kendrick Part III being smaller.

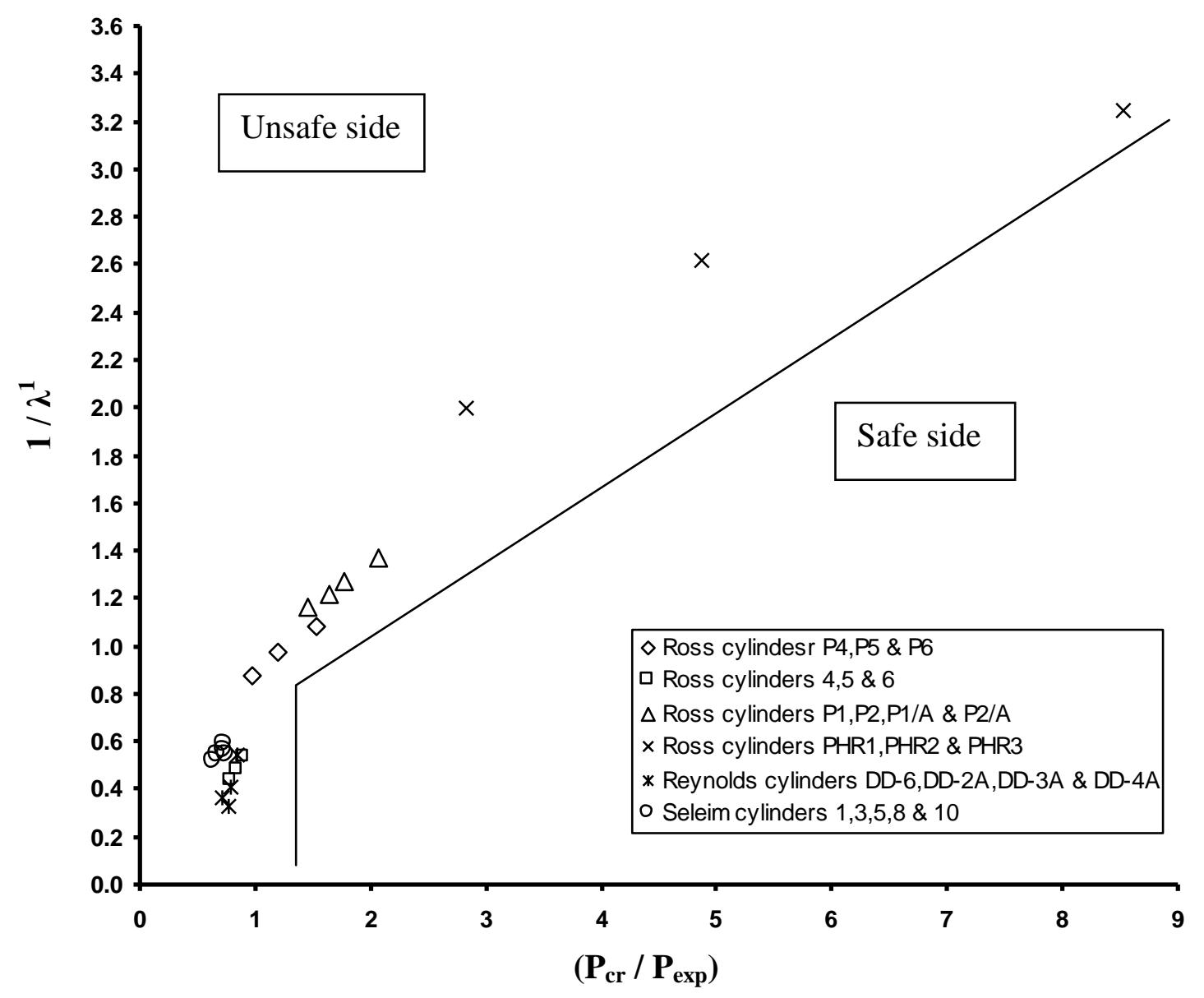

Figure 7: Design chart for the general instability of machined ring-stiffened cylinders based on Kendrick's Part III theory 
Kendrick's theories have been widely accepted and from the results of the present study, shown in Figures 6 and 7, it can be seen that comparisons between results from Kendrick Part I and III and experimentally obtained results is good. Even though Figures 6 and 7 are similar, both have been included because some designers prefer Kendrick Part I, while others prefer Kendrick Part III.

Buckling pressures from ANSYS were based on the finite element solution. With the use of ANSYS the boundary conditions could be adjusted using tools provided in the software. To simulate the exact situation of the experimental set-up, one end was completely fixed with all degrees of freedom set at zero, while the other end was left free and a thick end cap attached to this end. From Figures 6 to 8, it can be seen that the theoretical buckling pressures predicted by ANSYS were generally higher than for Kendrick Part I; in most cases, but only by a maximum of about $25 \%$.

The method for using the design charts is to first calculate the thinness ratio $\left(\lambda^{1}\right)$ and the theoretical buckling pressure $\left(\mathrm{P}_{\mathrm{cr}}\right)$ for the appropriate vessel. Then by applying the calculated value of the reciprocal thinness ratio to the appropriate design chart, to determine the PKD. The predicted buckling pressure $\left(\mathrm{P}_{\text {pred }}\right)$ can then be obtained by dividing the previously calculated theoretical buckling pressure, by the plastic knockdown factor. The design pressure $\left(\mathrm{P}_{\text {design }}\right)$ can then be obtained by dividing the predicted buckling pressure by a suitable factor (SF). The process is summarised as follows: Knowing $1 / \lambda^{1}$, PKD can be determined from either Figs 6 or 7 or 8 , as appropriate. $\mathrm{P}_{\text {pred }}=\mathrm{P}_{\mathrm{cr}} / \mathrm{PKD}$, where $\mathrm{P}_{\text {cr }}$ has already been calculated by the appropriate method. $\mathrm{P}_{\text {design }}=\mathrm{P}_{\text {pred }} / \mathrm{SF}$.

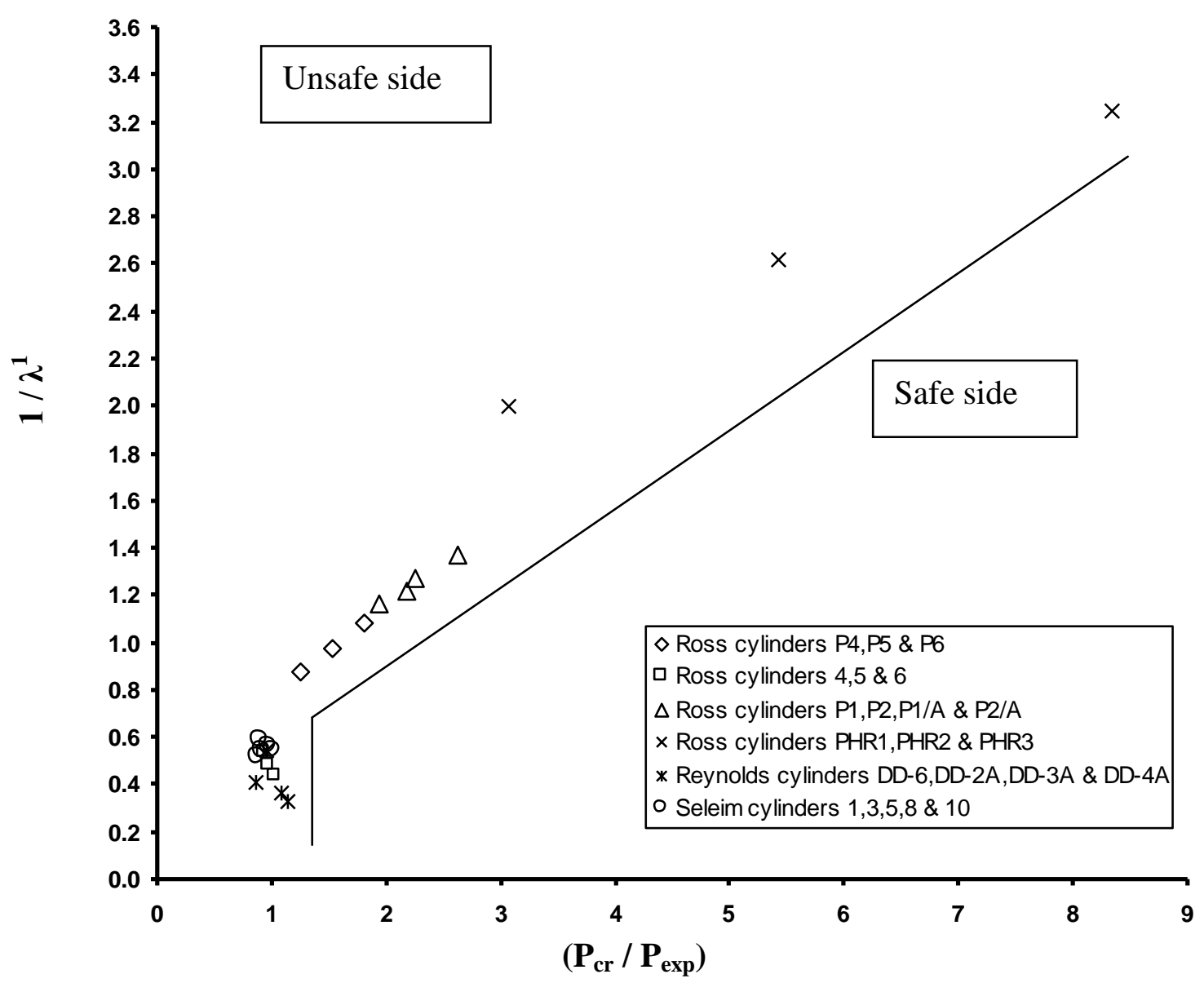

Figure 8: Design chart for the general instability of machined ring-stiffened cylinders using results from ANSYS 


\section{Conclusions and recommendations.}

An examination of the experimental procedure and theoretical investigations on twenty-two ringstiffened cylinders was carried out successfully. The main findings are listed as follows:

- Design charts were created successfully using analytical solutions based on Kendrick's Part I and Part III theories, and from ANSYS finite element analysis.

- Kendrick's theories show more reasonable and consistent results when compared with the experimental results, which therefore should be preferred to the results of ANSYS, especially because the Kendrick theories are much easier to use than ANSYS.

- Computational time spent obtaining results using ANSYS was in the ratio of about 1000:1 as compared with KENDRIC1 and KENDPT3, which employ Kendrick's analytical solutions.

- The design charts created during this project should prove useful to designers, even those for ANSYS, which showed differences with the analytical predictions, are useful.

Recommendations for further work:

- More experiments are needed, especially for lower thinness ratios, to improve and extend the design charts and to increase the accuracy of the predicted buckling pressures.

- The ANSYS results require further investigation. The results could be compared to exact Finite Element software such as RCONEBUR developed by Ross [1].

- The range of diameters of the models investigated was between $100.6 \mathrm{~mm}$ to $262.4 \mathrm{~mm}$. It is recommended that experimental and theoretical studies with larger diameter specimens be carried out and results combined with results from this investigation to produce improved design charts.

- The significance of the effect of boundary conditions on the inelastic buckling pressure of ring-stiffened circular cylinders needs to be studied further with a wide range of experimental and theoretical data.

\section{References.}

[1] Ross, C.T.F., Pressure Vessels: External Pressure Technology, Chichester, Horwood Publishing Limited, (2001), pp, 1-4, 284-87.

[2] Wikipedia, Online Encyclopaedia, http://en.wikipedia.org/wiki/Ocean http://en.wikipedia.org/wiki/Pressure

[3] Tokugawa, T., Model Experiments on the Elastic Stability of Closed and Cross-Stiffened Circular Cylinders under Uniform External Pressure, Proc. World Eng.ng. Congress, Tokyo, Vol. 29, Paper No. 651, (1929), pp. 249-79.

[4] Kendrick,S., The Buckling under External Pressure of Circular Cylindrical Shells with Evenly Spaced, Equal Strength Circular Ring-Frames-Part I, NCRE Report No. R.211, (1953). 
[5] Kendrick,S., The Buckling under External Pressure of Circular Cylindrical Shells with Evenly Spaced, Equal Strength Circular Ring-Frames-Part III, NCRE Report No. R.244, (1953).

[6] Kaminsky, E.L., General Instability of Ring-Stiffened Cylinders with Clamped Edges under External Pressure by Kendrick’s Method, DTMB Report No. 855, (July 1954).

[7] Nash, W.A., General Instability of Ring-Reinforced Shells Subject to Hydrostatic Pressure, Proc. $2^{\text {nd }}$ US National Congress on Applied Mechanics, (June 1954), pp. 359-68.

[8] Nash, W.A., Hydrostatically Loaded Structures, Pergamon, Oxford, U.K., (1995).

[9] Windenburg, D.F. \& Trilling, C., Collapse by Instability of Thin Cylindrical Shells Under External Pressure, Trans. ASME, 11, (1934), pp. 819-825.

[10] Ross, C.T.F., Pressure Vessels under External Pressure, Chapman \& Hall, U.K., (1990).

[11] Ross, C.T.F., Finite Element Methods in Engineering Science, Ellis Horwood, Chichester, U.K., (1990), pp. 13-14.

[12] Ross, C.T.F., Haynes, P., Seers, A \& Johns, T., Inelastic Buckling of Ring-Stiffened Circular Cylinders under Uniform External Pressure, Proc. Int. Conf. on Structural Dynamics and Vibrations, ASME, P.D. Vol. 70, (1995), pp. 207-215.

[13] Seleim, S.S. \& Roorda, J., Buckling Behaviour of Ring-Stiffened Cylinders, Experimental Study, J. Thin-Walled Structures,4, (1986), pp. 203-222.

[14] Reynolds, T.E. \& Blumenberg, W.F, General Instability of Ring-Stiffened Cylindrical Shells Subject to External Hydrostatic Pressure, DTMB Report No. 1324, (June 1959). 